\title{
A Schur complement approach to preconditioning sparse linear least-squares problems with some dense rows
}

\author{
Jennifer Scott ${ }^{1,2}$ (D) Miroslav Tůma ${ }^{3}$
}

Received: 11 April 2017 / Accepted: 10 January 2018 / Published online: 31 January 2018

(C) The Author(s) 2018. This article is an open access publication

\begin{abstract}
The effectiveness of sparse matrix techniques for directly solving largescale linear least-squares problems is severely limited if the system matrix $A$ has one or more nearly dense rows. In this paper, we partition the rows of $A$ into sparse rows and dense rows $\left(A_{s}\right.$ and $\left.A_{d}\right)$ and apply the Schur complement approach. A potential difficulty is that the reduced normal matrix $A_{s}^{T} A_{s}$ is often rank-deficient, even if $A$ is of full rank. To overcome this, we propose explicitly removing null columns of $A_{s}$ and then employing a regularization parameter and using the resulting Cholesky factors as a preconditioner for an iterative solver applied to the symmetric indefinite reduced augmented system. We consider complete factorizations as well as incomplete Cholesky factorizations of the shifted reduced normal matrix. Numerical experiments are performed on a range of large least-squares problems arising from practical applications. These demonstrate the effectiveness of the proposed approach when combined with either a sparse parallel direct solver or a robust incomplete Cholesky factorization algorithm.
\end{abstract}

Jennifer Scott was supported by EPSRC grant EP/M025179/1.

Miroslav Tüma was supported by projects 17-04150J and 18-12719S of the Grant Agency of the Czech Republic.

Jennifer Scott

jennifer.scott@stfc.ac.uk

Miroslav Tůma

mirektuma@karlin.mff.cuni.cz

1 STFC Rutherford Appleton Laboratory, Harwell Campus, Didcot, Oxfordshire, OX11 0QX, UK

2 School of Mathematical, Physical and Computational Sciences, University of Reading, Reading RG6 6AQ, UK

3 Department of Numerical Mathematics, Faculty of Mathematics and Physics, Charles

University, Prague, Czech Republic 
Keywords Large-scale linear least-squares problems · Dense rows · Augmented system · Schur complement · Iterative solvers · Preconditioning · Cholesky factorization $\cdot$ Incomplete factorizations

\section{Introduction}

We are interested in solving the linear least-squares (LS) problem

$$
\min _{x}\|A x-b\|_{2}
$$

where $A \in \mathfrak{R}^{m \times n}(m \geq n)$ and $b \in \mathfrak{R}^{m}$. The solution $x$ satisfies the $n \times n$ normal equations

$$
C x=A^{T} b, \quad C=A^{T} A,
$$

where provided $A$ has full column rank, the normal matrix $C$ is symmetric and positive definite. Our focus is on the case where the system matrix $A$ is large and sparse but has a number of "dense" rows (rows that contain significantly more entries than the other rows, although the number of entries in each such row may be less than $n$ ). Just a single dense row is sufficient to cause catastrophic fill in $C$ and thus for the factors of a Cholesky or QR factorization to be dense. In practice, for largescale problems, this means that it may not be possible to use a direct solver because the memory demands can be prohibitive. Moreover, if an incomplete factorization is used as a preconditioner for an iterative solver such as LSQR [37, 38] or LSMR [13] applied to (1.1), the error in the factorization can be so large as to prohibit its effectiveness as a preconditioner; this was recently observed in the study by Gould and Scott [20]. The effect of dense rows has long been recognised as a fundamental difficulty in the solution of sparse least-squares problems (see, for example, $[2,5,8,14$, 17, 48-51]).

Let us assume that the rows of $A$ are partitioned into two parts: rows that are sparse and those that are considered dense. We also assume conformal partitioning of the right-hand side vector $b$ as follows:

$$
A=\left(\begin{array}{c}
A_{s} \\
A_{d}
\end{array}\right), A_{s} \in R^{m_{s} \times n}, A_{d} \in R^{m_{d} \times n}, b=\left(\begin{array}{c}
b_{s} \\
b_{d}
\end{array}\right), b_{s} \in R^{m_{s}}, b_{d} \in R^{m_{d}},
$$

with $m=m_{s}+m_{d}, m_{s} \geq n$ and $m_{d} \geq 1$ (in general, $m_{s} \gg m_{d}$ ). Problem (1.1) then becomes

$$
\min _{x}\left\|\left(\begin{array}{l}
A_{s} \\
A_{d}
\end{array}\right) x-\left(\begin{array}{l}
b_{s} \\
b_{d}
\end{array}\right)\right\|_{2} .
$$

A number of approaches to tackling such systems have been proposed. For example, George and Heath [14] temporarily discard $A_{d}$ to avoid severe fill-in in their Givens rotation-based orthogonal factorization of $A_{s}$. They then employ an updating scheme to allow for the effect of these rows. A completely general updating algorithm for the constrained least-squares problem based on $\mathrm{QR}$ decomposition and direct elimination is given by Björck [7], while Avron et al. [5] propose dropping dense rows before the QR factorization is performed and using the resulting $R$ factor as a preconditioner for LSQR. Most recently, Scott and Tưma [48] process rows that are identified as 
dense separately within a conjugate gradient method using an incomplete factorization preconditioner combined with the factorization of a dense matrix of size equal to the number of dense rows.

Solving (1.4) is equivalent to solving the larger $(m+n) \times(m+n)$ augmented system

$$
\left(\begin{array}{ccc}
I_{m_{s}} & & A_{s} \\
& I_{m_{d}} & A_{d} \\
A_{s}^{T} & A_{d}^{T} & 0
\end{array}\right)\left(\begin{array}{c}
r_{s} \\
r_{d} \\
x
\end{array}\right)=\left(\begin{array}{c}
b_{s} \\
b_{d} \\
0
\end{array}\right)
$$

where

$$
r=\left(\begin{array}{l}
r_{s} \\
r_{d}
\end{array}\right)=\left(\begin{array}{l}
b_{s} \\
b_{d}
\end{array}\right)-\left(\begin{array}{l}
A_{s} \\
A_{d}
\end{array}\right) x
$$

is the residual vector. Here and elsewhere, $I_{k}$ denotes the $k \times k$ identity matrix. System (1.5) is symmetric indefinite and so, if there is sufficient memory available, a sparse direct solver that incorporates numerical pivoting for stability can be used (well-known examples include MA57 [12] and HSL_MA97 [23] from the HSL mathematical software library [24], MUMPS [31] and WSMP [53]). Employing a general-purpose sparse solver ignores the block structure, although its use of a sparsity-preserving ordering (such as a variant of minimum degree or nested dissection) will tend to lead to the dense rows being eliminated last [11].

An alternative approach is to eliminate $r_{s}$ to reduce the problem from a 3-block saddle-point system to a 2-block system of order $\left(n+m_{d}\right) \times\left(n+m_{d}\right)$ that can be written in the form

$$
K\left(\begin{array}{c}
x \\
r_{d}
\end{array}\right)=\left(\begin{array}{c}
-A_{s}^{T} b_{s} \\
b_{d}
\end{array}\right), \quad K=\left(\begin{array}{cc}
-C_{s} & A_{d}^{T} \\
A_{d} & I_{m_{d}}
\end{array}\right),
$$

where the $n \times n$ matrix $C_{s}=A_{s}^{T} A_{s}$ is termed the reduced normal matrix. We refer to (1.6) as the reduced augmented system. The reduced augmented matrix $K$ can be factorized using a sparse indefinite solver, but this would again ignore the block structure. Instead, we use the so-called Schur complement method (see, for example, $[15,30,42]$ and Section 2 below) that exploits the structure by performing a sparse Cholesky factorization of $A_{s}^{T} A_{s}$, forming an $m_{d} \times m_{d}$ dense Schur complement matrix and factorizing it using a dense Cholesky factorization; using the sparse and dense factors to solve a number of triangular systems completes the solution process. This has the advantage of using Cholesky factorizations that, because they do not involve numerical pivoting, are more efficient (especially in parallel) than an indefinite factorization. Moreover, it can be easily incorporated into the normal equations approach.

We observe that the reduced augmented matrix $K$ is symmetric quasi-definite (SQD). Vanderbei [52] shows that SQD matrices are strongly factorisable and this allows general permutations to be used [35]. Recent work has shown that some classes of permutations can be beneficial and underlying theory has been developed that helps to find such permutations for deriving incomplete factorization preconditioners [21, 34, 47]. An interesting stability analysis of the factorization of SQD matrices that connects the stability with the effective condition number is given in [16] (see also [18]). Furthermore, analysis of a symmetric indefinite factorization based on the related generalized QR factorization in [33] points out that conditioning 
of the principal leading submatrices may be determined by other factors such as the number of sign changes in the diagonal of the signed factorization. In this paper, we maintain the block structure and this means that the stability is predetermined by the splitting into sparse and dense rows. Note that the results in [48] show that iterative methods are very sensitive to keeping all the dense rows separate and not mixing any of them with the other rows.

Another possibility is to use an iterative solver such as LSQR [37, 38] that has the advantage of avoiding the construction of the dense matrices that are involved in the Schur complement approach (see (2.2) and (2.3) below). However, in many cases, the LS problems are hard to solve and a preconditioner is required to achieve acceptable convergence.

In practice, even if $A$ is of full rank, $A_{s}$ is often rank-deficient (indeed, it may have null columns). In this case, a Cholesky factorization of $A_{s}^{T} A_{s}$ will break down. To overcome this, we propose removing null columns and employing a regularization parameter and using the resulting Cholesky factors as a preconditioner for an iterative solver applied to the reduced augmented system. For large problems, even if $A_{s}$ is sparse, memory limitations can mean that it is not possible to use a sparse direct solver. Thus, we consider using incomplete Cholesky factorizations combined with an iterative solver.

The outline of the rest of the paper is as follows. In Section 2, we recall the Schur complement approach and, in particular, we look at regularization and propose using the factors of the reduced regularized matrix $A_{s}^{T} A_{s}+\alpha I$ to obtain a block preconditioner. Use of a limited memory incomplete Cholesky factorization is also discussed. Section 3 introduces our numerical experiments. Computational results for complete and incomplete Cholesky factorizations are given in Sections 4 and 5, respectively. Concluding remarks are made in Section 6.

\section{Schur complement method}

\subsection{Schur complement method with direct solvers}

As already observed, an alternative to applying a direct solver to either the (dense) normal equations or the augmented system (1.5) is to solve the reduced augmented system (1.6). Provided $A_{s}$ has full column rank, $C_{S}$ is symmetric positive definite and, if the partitioning (1.3) is such that all the rows of $A_{s}$ are sparse, $C_{S}$ is generally significantly sparser than the original normal matrix $C$. Let $C_{s}=L_{S} L_{S}^{T}$ be the Cholesky factorization of $C_{s}$. Using this yields a block factorization

$$
K=\left(\begin{array}{ll}
L_{s} & \\
B_{d} & I_{m_{d}}
\end{array}\right)\left(\begin{array}{ll}
-I_{n} & \\
& S_{d}
\end{array}\right)\left(\begin{array}{cc}
L_{s}^{T} & B_{d}^{T} \\
& I_{m_{d}}
\end{array}\right),
$$

where $B_{d}$ and the Schur complement matrix $S_{d}$ are given by

$$
\begin{aligned}
L_{s} B_{d}^{T} & =-A_{d}^{T} \\
S_{d} & =I_{m_{d}}+B_{d} B_{d}^{T} .
\end{aligned}
$$


Since $L_{s}$ is lower triangular, solving (2.2) for $B_{d}^{T}$ is straightforward. $B_{d}$ will normally be dense and hence $S_{d}$ will be dense and symmetric positive definite. Once we have $L_{s}, B_{d}$ and $S_{d}$, we can solve (1.6) by solving

$$
\left(\begin{array}{ll}
-L_{s} & \\
-B_{d} & S_{d}
\end{array}\right)\left(\begin{array}{l}
y_{s} \\
y_{d}
\end{array}\right)=\left(\begin{array}{c}
-A_{s}^{T} b_{s} \\
b_{d}
\end{array}\right),
$$

followed by

$$
\left(\begin{array}{cc}
L_{s}^{T} & B_{d}^{T} \\
& I_{m_{d}}
\end{array}\right)\left(\begin{array}{c}
x \\
r_{d}
\end{array}\right)=\left(\begin{array}{c}
y_{s} \\
y_{d}
\end{array}\right) .
$$

With $r_{d}=y_{d}$, this requires us to solve

$$
\begin{aligned}
L_{s} y_{s} & =A_{s}^{T} b_{s}, \\
S_{d} r_{d} & =b_{d}+B_{d} y_{s}, \\
L_{s}^{T} x & =y_{s}-B_{d}^{T} r_{d} .
\end{aligned}
$$

Again, (2.6) and (2.8) are triangular systems and hence straightforward to solve. Solving (2.7) requires Cholesky factorization of the $m_{d} \times m_{d}$ dense matrix $S_{d}$. Observe that in the case of a single dense row $\left(m_{d}=1\right)$ this is trivial. In general, the LAPACK routine _potrf can be used to factorize $S_{d}$ and then routine_potrs employed to solve (2.7). Note also that $B_{d}$ need not be computed explicitly. Rather, $S_{d}$ may be computed implicitly as $I_{m_{d}}+A_{d} L_{s}^{-T} L_{s}^{-1} A_{d}^{T}$, while $z=B_{d} y_{s}$ may be computed by solving $L_{s} w=y_{s}$ and then $z=-A_{d} w$ and $z=-B_{d}^{T} r_{d}$ may be obtained by solving $L_{s} z=A_{d}^{T} r_{d}$.

\subsection{Scaling and stability}

Poorly scaled entries in $A_{s}$ may result in block elimination of the first $m_{s}$ rows and columns of (1.5) being unstable. To overcome this, we prescale $A$ so that the entries of the scaled $A$ are small relative to 1 . Thus, we scale $A$ by normalising each column by its 2 -norm. That is, we replace $A$ by $A D$, where $D$ is the diagonal matrix with entries $D_{j j}=1 /\left\|A e_{j}\right\|_{2}\left(e_{j}\right.$ denotes the $j$-th unit vector). The entries of $A D$ are all less than one in absolute value. Elimination of the first $m_{s}$ rows and columns is thus stable (the pivots can be chosen in order from the main diagonal and they satisfy the criteria for complete pivoting). However, this does not guarantee stability of the next step. The diagonal entries of the factor $L_{s}$ of the positive definite $C_{s}$ can be small, leading to large entries in $B_{d}$ and hence large entries in $S_{d}$.

If instability is detected, then a general-purpose symmetric indefinite sparse solver that incorporates numerical pivoting can be applied to solve the reduced augmented system (1.6). Whilst this offers a robust approach, it has the disadvantage that the block structure within (1.6) is not exploited. Furthermore, if $A_{s}$ is fixed and the interest lies in adding new rows $A_{d}$, the factorization must be redone in its entirety for each $A_{d}$. Thus, in Section 2.4, we propose an alternative approach to maintain stability.

We assume throughout the remainder of our discussion and in all our numerical experiments that $A$ has been prescaled, but we omit $D$ to simplify the notation. 


\subsection{Removal of null columns}

In practice, when $A$ is partitioned, the sparse part $A_{s}$ often contains a (small) number of null columns. It is possible to remove these columns explicitly, as we now show. Let $A$ have full column rank and assume $A_{s}$ has $n_{2}$ null columns with $n_{2} \ll n$. Assuming these columns are permuted to the end, we can split $A$ into the form

$$
A=\left(\begin{array}{ll}
A_{1} & A_{2}
\end{array}\right) \equiv\left(\begin{array}{cc}
A_{s_{1}} & 0 \\
A_{d_{1}} & A_{d_{2}}
\end{array}\right)
$$

with $A_{1} \in R^{m \times n_{1}}$ and $A_{2} \in R^{m \times n_{2}}\left(n=n_{1}+n_{2}\right)$. The following result from [48] shows that the solution of the least-squares problem can be expressed as a combination of partial solutions.

Lemma 2.1 Let the columns of $A$ be split as in (2.9) and let $z \in R^{n_{1}}$ and $W \in$ $R^{n_{1} \times n_{2}}$ be the solutions to the problems

$$
\min _{z}\left\|A_{1} z-b\right\|_{2} \text { and } \min _{W}\left\|A_{1} W-A_{2}\right\|_{F},
$$

respectively. Assuming $A_{1}$ is of rank $n_{1}$, the solution $x=\left(\begin{array}{l}x_{1} \\ x_{2}\end{array}\right)$ of the least-squares problem (1.1) with its splitting consistent with (2.9) (that is, $x_{1} \in R^{n_{1}}, x_{2} \in R^{n_{2}}$ ) is given by

$$
\left(\begin{array}{l}
x_{1} \\
x_{2}
\end{array}\right)=\left(\begin{array}{c}
z-W x_{2} \\
x_{2}
\end{array}\right)
$$

with

$$
x_{2}=\left(A_{2}^{T}\left(A_{2}-A_{1} W\right)\right)^{-1} A_{2}^{T}\left(b-A_{1} z\right) .
$$

The least-squares problems (2.10) again have $m_{d}$ dense rows and thus can be solved using the Schur complement method as described in (2.1) - (2.8). In this case, we use the subscripts $s_{1}$ and $d_{1}$ for $C_{s_{1}}, L_{s_{1}}, S_{d_{1}}$ and $B_{d_{1}}$ to emphasize we are working with $A_{1}$, which has fewer columns than $A$. The factorizations of the reduced normal matrix $C_{s_{1}}=A_{s_{1}}^{T} A_{s_{1}}$ and the Schur complement matrix $S_{d_{1}}$ needed to compute $z$ can be used to solve for $W$. For the latter, if $A_{2 j}$ denotes a column $j$ of $A_{d_{2}}\left(1 \leq j \leq n_{2}\right)$, the right-hand side in (2.4) based on $A_{1}$ becomes $\left(\begin{array}{c}0 \\ A_{2 j}\end{array}\right)$ and thus (2.6) - (2.8) reduce to solving systems of the form $S_{d_{1}} R_{d j}=A_{2 j}$ and $L_{s_{1}}^{T} W_{j}=-B_{d_{1}}^{T} R_{d j}$. If we are using direct solvers that allow for solves with multiple right-hand sides, we can perform these solves for all the $R_{d j}$ and $W_{j}$ simultaneously with solving (2.7) and (2.8). Thus, as solving for multiple right-hand sides is generally significantly less expensive than repeatedly solving for a single right-hand side (since multiple right-hand sides allow the use of level 3 BLAS), the extra solves add only a small overhead. Furthermore, using (2.9), (2.12) reduces to

$$
x_{2}=\left(A_{d_{2}}^{T}\left(A_{d_{2}}-A_{d_{1}} W\right)\right)^{-1} A_{d_{2}}^{T}\left(b_{d}-A_{d_{1}} z\right) .
$$

Forming and then solving with $A_{d_{2}}^{T}\left(A_{d_{2}}-A_{d_{1}} W\right)$ is inexpensive provided $n_{2} \ll n$. 


\subsection{Regularization and preconditioning}

While Lemma 2.1 provides a way of removing null columns, it is possible that even if $A$ has full column rank, $A_{s}$ (or $A_{s_{1}}$ after the removal of null columns) is rank-deficient (or is close to rank-deficient). To simplify notation, in our discussion, we use $A_{s}$ but this may be replaced by $A_{s_{1}}$ if $A_{s}$ contains null columns. If $A_{s}$ is rank-deficient, the reduced normal matrix $C_{S}$ is positive semidefinite and a Cholesky factorization breaks down (that is, a very small or a non-positive pivot is encountered). If breakdown occurs, we employ a shift $\alpha>0$ and compute a Cholesky factorization of the shifted matrix

$$
C_{s}(\alpha)=A_{s}^{T} A_{s}+\alpha I_{n} .
$$

The shift $\alpha$ is also referred to as a Tikhonov regularization parameter. The choice of $\alpha$ should be related to the smallest eigenvalue of $A_{s}^{T} A_{s}$, but this information is not readily available. Clearly, it is always possible to find an $\alpha>0$ so that the factorization does not breakdown; if the initial choice $\alpha$ is too small, it may be necessary to restart the factorization more than once, increasing $\alpha$ on each restart until breakdown is avoided. Use of a shift was discussed by Lustig et al. [28] (see also [1, 2]). It was observed that careful and often substantial use of iterative refinement to compute each column of $B_{d}^{T}$ was required. However, we adopt a different approach in which we use the factorization of (2.14) to obtain a preconditioner for the system (1.6). Note that this regularization that shifts all the diagonal entries could be considered as an over-regularization of the system since we could regularize only those entries that cause the factorization to break down. Our strategy is based on experimental evidence that shows that for incomplete Cholesky (IC) factorizations, it is better to use a global shift as introduced by Manteuffel [29] rather than a local shifting strategy [6, 45]. Contemporary incomplete factorizations such as that described in [45] increase and decrease the shift automatically (see also [27]).

With $\alpha>0$ in (2.14), we approximate the solution of (1.6) by changing $K$ to

$$
K(\alpha)=\left(\begin{array}{cc}
-C_{s}(\alpha) & A_{d}^{T} \\
A_{d} & I_{m_{d}}
\end{array}\right) .
$$

Thus, the computed value of the least-squares objective may differ from the optimum for the original problem. Having solved the regularized problem, we want to recover the solution of the original problem. Following Scott [44], we propose doing this by using the factors of $K(\alpha)$ as a preconditioner for an iterative method applied to (1.6).

Let the Cholesky factorization of $C_{s}(\alpha)$ be $L_{s}(\alpha) L_{s}(\alpha)^{T}$. For $\alpha>0$, this is an approximate factorization of $C_{s}$, that is, $C_{s} \approx L_{s}(\alpha) L_{s}(\alpha)^{T}$. More generally, let

$$
C_{s} \approx \tilde{L}_{s} \tilde{L}_{s}^{T},
$$

where $\tilde{L}_{s}$ is lower triangular. We are interested in the case $\tilde{L}_{s}=L_{s}(\alpha)$ but our main focus is where $\tilde{L}_{s}$ is an incomplete Cholesky (IC) factor, that is, one that contains fewer entries than occur in a complete factorization. For very large systems, computing and factorizing $C_{S}\left(\right.$ or $\left.C_{S}(\alpha)\right)$ is prohibitively expensive in terms of memory 
and/or computational time. Over the last 50 or more years, IC factorizations have been an important tool in the armoury of preconditioners for the numerical solution of large sparse symmetric positive-definite linear systems of equations; for an introduction and overview see, for example, [6, 40,46] and the long lists of references therein. Here, we consider preconditioning the symmetric indefinite system (1.6) using a factorization of the form (2.15) and exploiting the block structure of (1.6).

The right-preconditioned reduced augmented system is

$$
K M^{-1}\left(\begin{array}{c}
w_{s} \\
w_{d}
\end{array}\right)=\left(\begin{array}{c}
-A_{s}^{T} b_{s} \\
b_{d}
\end{array}\right), \quad M\left(\begin{array}{c}
x \\
r_{d}
\end{array}\right)=\left(\begin{array}{c}
w_{s} \\
w_{d}
\end{array}\right),
$$

where $M$ is the chosen preconditioner. Using (2.15), we obtain an indefinite preconditioner $M$ given by

$$
M=\left(\begin{array}{ll}
\tilde{L}_{s} & \\
\tilde{B}_{d} & I_{m_{d}}
\end{array}\right)\left(\begin{array}{ll}
-I_{n} & \\
& \tilde{S}_{d}
\end{array}\right)\left(\begin{array}{cc}
\tilde{L}_{s}^{T} & \tilde{B}_{d}^{T} \\
& I_{m_{d}}
\end{array}\right),
$$

where $\tilde{B}_{d}$ and $\tilde{S}_{d}$ are given by (2.2) and (2.3) with the complete factor $L_{s}$ replaced by the incomplete one $\tilde{L}_{s}$, that is,

$$
\begin{aligned}
\tilde{L}_{s} \tilde{B}_{d}^{T} & =-A_{d}^{T} \\
\tilde{S}_{d} & =I_{m_{d}}+\tilde{B}_{d} \tilde{B}_{d}^{T} .
\end{aligned}
$$

Applying this preconditioner requires a number of steps that are analogous to (2.6)(2.8). In particular, a dense $m_{d} \times m_{d}$ symmetric positive-definite system of the form $\tilde{S}_{d} y_{d}=u_{d}$ must be solved. We again assume that LAPACK may be used. If $m_{d}$ is so large that this is too expensive, an incomplete factorization of $\tilde{S}_{d}$ could be used. Algorithm 1 outlines the steps required for each application of the preconditioner. We see that it involves a triangular solve with $\tilde{L}_{s}$ and with $\tilde{L}_{s}^{T}$, calls to the BLAS routine _gemv for steps 2 and 4, and triangular solves using the factors of $\tilde{S}_{d}$. As before, $\tilde{B}_{d}$ need not be held explicitly but can be applied implicitly using $\tilde{B}_{d}^{T}=-\tilde{L}_{s}^{-1} A_{d}^{T}$.

\footnotetext{
$\overline{\text { Algorithm } 1 \text { Application of the block factorization preconditioner, that is, compute }}$ $y=M^{-1} z$.
}

Input: $\tilde{L}_{s}, \tilde{B}_{d}$, the Cholesky factors of $\tilde{S}_{d}$, and the vector $z=\left(\begin{array}{c}z_{s} \\ z_{d}\end{array}\right)$.

Output: $y=\left(\begin{array}{l}y_{s} \\ y_{d}\end{array}\right)=M^{-1} z$.

1: Solve $\tilde{L}_{s} u_{s}=-z_{s}$.

2: Compute $u_{d}=z_{d}+\tilde{B}_{d} u_{s}$.

3: Use the Cholesky factors of $\tilde{S}_{d}$ to solve $\tilde{S}_{d} y_{d}=u_{d}$.

4: Form $u_{s}=u_{s}-\tilde{B}_{d}^{T} y_{d}$.

5: Solve $\tilde{L}_{s}^{T} y_{s}=u_{s}$. 
As the preconditioner (2.17) is indefinite, it needs to be used with a general nonsymmetric iterative method such as GMRES [41]. We can obtain a positive-definite preconditioner for use with MINRES [36] by replacing $M$ by

$$
|M|=\left(\begin{array}{ll}
\tilde{L}_{s} & \\
\tilde{B}_{d} & I_{m_{d}}
\end{array}\right)\left(\begin{array}{ll}
I_{n} & \\
& \tilde{S}_{d}
\end{array}\right)\left(\begin{array}{cc}
\tilde{L}_{s}^{T} & \tilde{B}_{d}^{T} \\
& I_{m_{d}}
\end{array}\right),
$$

In Algorithm 1 , steps 1 and 2 are then replaced by 1 . Solve $\tilde{L}_{s} u_{s}=z_{s}$ and 2. Compute $u_{d}=z_{d}-\tilde{B}_{d} u_{s}$. MINRES has the advantage over GMRES of using short recurrences that limit the storage requirements.

Many different IC factorizations have been proposed. Although they may be considered to be general-purpose, most are best-suited to solving particular classes of problems. For example, level-based methods are often appropriate for systems with underlying structure, such as from finite element or finite difference applications. Here, we use the limited memory approach of Scott and Tüma [45, 46] that has been shown in [20] to result in effective preconditioners for a wide range of least-squares problems. The basic scheme employs a matrix factorization of the form

$$
C_{s} \approx\left(\tilde{L}_{s}+\tilde{R}_{s}\right)\left(\tilde{L}_{s}+\tilde{R}_{s}\right)^{T},
$$

where $\tilde{L}_{s}$ is the lower triangular matrix with positive diagonal entries that is used for preconditioning and $\tilde{R}_{S}$ is a strictly lower triangular matrix with small entries that is used to stabilize the factorization process but is then discarded (it is not used as part of the preconditioner). The user specifies the maximum number of entries in each column of $\tilde{L}_{S}$ and $\tilde{R}_{S}$. At each step $j$ of the incomplete factorization process, the largest entries are kept in column $j$ of $\tilde{L}_{s}$, the next largest are kept in column $j$ of $\tilde{R}_{S}$, and the remainder (the smallest entries) are dropped. In practice, $C_{S}$ is optionally preordered and scaled and, if necessary, shifted to avoid breakdown of the factorization (which occurs if a non-positive pivot is encountered) [29].

\section{Numerical experiments}

In this section, we present numerical results to illustrate potential of the Schur complement approach and, in particular, demonstrate that it allows us to solve some problems that are intractable if dense rows are ignored. Results are included for direct solvers and for iterative solvers that can be used to solve very large problems.

\subsection{Test environment}

The characteristics of the machine used to perform our tests are given in Table 1.

All software is written in Fortran and all reported timings are elapsed times in seconds. In our experiments, we employ the Cholesky sparse direct solver HSL_MA8 7 [22] for positive-definite systems and HSL_MA97 [23] for general sparse symmetric indefinite systems; both employ OpenMP and are run in parallel, using four processors. Both solvers are run with a nested dissection ordering [26]. Sparse matrix-vector products required by the iterative solvers are performed in parallel using the Intel Mathematics Kernel Library (MKL) routines; no attempt is made to parallelize the 
Table 1 Test machine characteristics

$\begin{array}{ll}\text { CPU } & \text { Two Intel Xeon E5620 quadcore processors } \\ \text { Memory } & 24 \mathrm{~GB} \\ \text { Compiler } & \text { gfortran version 4.8.4 with options -O3 -fopenmp } \\ \text { BLAS } & \text { Intel MKL }\end{array}$

iterative methods themselves. In each test, we impose a time limit of $600 \mathrm{~s}$ per problem and for the iterative methods, the number of iterations is limited to 100,000.

Following Gould and Scott [20], we want the computed residual $r$ to satisfy either $\left\|r_{k}\right\|_{2}<\delta_{1}$ or

$$
\text { ratio }(r)<\delta_{2} \quad \text { with } \quad \text { ratio }(r)=\frac{\left\|A^{T} r\right\|_{2} /\|r\|_{2}}{\left\|A^{T} b\right\|_{2} /\|b\|_{2}} .
$$

We set the tolerances $\delta_{1}$ and $\delta_{2}$ to $10^{-8}$ and $10^{-6}$, respectively, and in our experiments, the right-hand side $b$ is taken to be the vector of 1 's. We use rightpreconditioned restarted GMRES with the restart parameter set to 500, and in some experiments, we also report on using preconditioned MINRES. Since the iterative solver is applied to the reduced augmented system matrix $K$, the stopping criterion is applied to $K$. With the available implementations of GMRES and MINRES, it is not possible during the computation to check whether (3.1) is satisfied; this can only be checked once the solver has terminated. Instead, we use the scaled backward error

$$
\frac{\left\|K\left(\begin{array}{c}
x^{(k)} \\
r_{d}^{(k)}
\end{array}\right)-\left(\begin{array}{c}
-A_{s}^{T} b_{s} \\
b_{d}
\end{array}\right)\right\|_{2}}{\left\|\left(\begin{array}{c}
-A_{s}^{T} b_{s} \\
b_{d}
\end{array}\right)\right\|_{2}}<\tilde{\delta},
$$

where $\left(\begin{array}{l}x^{(k)} \\ r_{d}^{(k)}\end{array}\right)$ is the computed solution of (1.6) on the $k$ th step. In our experiments, we set $\tilde{\delta}=10^{-7}$. With this choice, in most of our experiments, (3.1) is satisfied with $\delta_{2}=10^{-6}$.

\subsection{Test set 1}

Our test problems are taken from the CUTEst linear programme set [19] and the University of Florida Sparse Matrix Collection [9]. In each case, the matrix is "cleaned" (duplicates are summed, out-of-range entries and explicit zeros are removed along with any null rows or columns). In our experiments, we use the following definition for a dense row of $A$ : given $\rho(0<\rho \leq 1)$, row $i$ of $A$ is defined to be dense if the percentage of entries in row $i$ is at least $\rho$.

Our first test set is given in Table 2. The problems were chosen because they have at least one row that is more than $10 \%$ dense. They are also difficult problems to solve (see [20]); at least three of the problems are rank-deficient. An estimate of the rank was computed by running the sparse symmetric indefinite solver HSL_MA97 on the 
Table 2 Statistics for test set 1

\begin{tabular}{lllllllllllll}
\hline Problem & $m$ & $n$ & $n n z(A)$ & nullity & rdensity $(A)$ & $m_{10}$ & $m_{20}$ & $m_{30}$ & $m_{40}$ & $m_{50}$ & $\operatorname{density}(C)$ \\
\hline Trec14 & 15,904 & 3159 & $2,872,265$ & 14 & 0.791 & 2664 & 1232 & 649 & 346 & 150 & $9.32 \times 10^{-1}$ \\
Maragal_6 & 21,251 & 10,144 & 537,694 & 516 & 0.586 & 68 & 68 & 30 & 21 & 0 & $7.49 \times 10^{-1}$ \\
Maragal_7 & 46,845 & 26,525 & $1,200,537$ & 2046 & 0.360 & 85 & 43 & 21 & 0 & 0 & $3.10 \times 10^{-1}$ \\
sCsd8-2r & 60,550 & 8650 & 190,210 & 0 & 0.100 & 40 & 0 & 0 & 0 & 0 & $5.22 \times 10^{-2}$ \\
PDE1 & 271,792 & 270,595 & 990,587 & - & 0.670 & 1 & 1 & 1 & 1 & 1 & - \\
12month1 & 872,622 & 12,471 & $22,624,727$ & - & 0.274 & 284 & 4 & 0 & 0 & 0 & $6.87 \times 10^{-1}$ \\
\hline
\end{tabular}

$m, n$ and $n n z(A)$ are the number of rows and columns and nonzeros in $A$. nullity is the estimated deficiency in the rank of $A, \operatorname{rdensity}(A)$ is the largest ratio of number of nonzeros in a row of $A$ to $n$ over all rows, $m_{j}(j=10,20,30,40,50)$ is the number of rows of $A$ with at least $j \%$ entries, and density $(C)$ is the ratio of the number of entries in $C$ to $n^{2}$. - denotes insufficient memory to compute the statistic

augmented system (1.5) (with the pivot threshold parameter set to 0.5); for problems 12 month 1 and $\mathrm{PDE} 1$, there was insufficient memory to do this.

In Table 3, we report the effects of varying the parameter $\rho$ that controls which rows are classified as dense. Increasing $\rho$ reduces the number $m_{d}$ of dense rows and the number $n_{2}$ of null columns in $A_{s}$ but increases the density of the reduced normal matrix $C_{s}$. Problem PDE1 has only one row that is classified as dense for

Table 3 The effects of varying the row density parameter $\rho$ on the number $m_{d}$ of rows that are classed as dense, the number $n_{2}$ of null columns in $A_{s}$, and the density of $C_{s}$ (the ratio of the number of entries in $C_{s}$ to $n^{2}$ )

\begin{tabular}{lllllll}
\hline Identifier & $m$ & $n$ & $\rho$ & $m_{d}$ & $n_{2}$ & $\operatorname{density~}\left(C_{s}\right)$ \\
\hline Trec14 & \multirow{2}{*}{15,904} & \multirow{2}{*}{3159} & 0.005 & 12,643 & 0 & $2.38 \times 10^{-2}$ \\
& & & 0.010 & 9676 & 0 & $8.52 \times 10^{-2}$ \\
& & & 0.050 & 4467 & 0 & $6.17 \times 10^{-1}$ \\
& & & 0.100 & 2664 & 0 & $8.31 \times 10^{-1}$ \\
Maragal_6 & 21,251 & \multirow{2}{*}{10,144} & 0.005 & 2923 & 759 & $6.22 \times 10^{-4}$ \\
& & & 0.010 & 823 & 3 & $1.93 \times 10^{-2}$ \\
& & & 0.100 & 68 & 2 & $5.49 \times 10^{-2}$ \\
Maragal_7 & 46,845 & 26,525 & 0.001 & 4668 & 2352 & $2.15 \times 10^{-4}$ \\
& & & 0.005 & 687 & 6 & $9.02 \times 10^{-3}$ \\
& & & 0.010 & 108 & 6 & $1.70 \times 10^{-2}$ \\
SCsd8-2x & 60,550 & 8650 & 0.050 & 50 & 5 & $1.78 \times 10^{-2}$ \\
& & & 0.100 & 40 & 0 & $1.39 \times 10^{-3}$ \\
PDE1 & 271,792 & 270,595 & 0.660 & 1 & 0 & $4.52 \times 10^{-2}$ \\
12month1 & 872,622 & 12,471 & 0.010 & 43,951 & 387 & $1.10 \times 10^{-1}$ \\
& & & 0.050 & 3641 & 17 & $5.66 \times 10^{-1}$ \\
& & & 0.100 & 284 & 3 & $6.56 \times 10^{-1}$ \\
\hline
\end{tabular}


$\rho \in[0.001,0.66]$. We see that for 12 month 1 and Trec $14, \rho$ has to be very small for $C_{s}$ to be sparse but, in this case, $m_{d}$ is large compared to $m$. For the Maragal problems, $C_{s}$ is highly sparse if approximately $10 \%$ of the rows are classified as dense.

\subsection{Test set 2}

For our second test set, we take some of the CUTEst and UFL examples that do not initially contain dense rows and append some rows. This allows us to explore the effect of varying the number of dense rows as well as the density of these rows. The problems are listed in Table 4; these problems are all of full rank. The pattern of each appended row is generated randomly with the requested density and the values of the entries are random numbers in $[-1,1]$.

For our solvers, the number of entries $n n z(C)$ in the normal matrix $C$ can be at most huge $(1)\left(\approx 2 \times 10^{9}\right)$, where huge is the Fortran intrinsic function. If we add a single row with density $\rho \geq 0.1$ to each of the matrices $A_{s}$ in the lower part of Table 4 then $n n z(C)$ exceeds this limit. Thus for these examples and our current software, we cannot use any approach that requires the normal matrix to be computed.

\section{Direct solver results}

Our first experiments look at the effectiveness of the Schur complement approach using the Cholesky direct solver HSL_MA87 to factorize the reduced normal matrix $C_{S}$. We compare this with using HSL_MA87 to solve the original normal matrix $C$ (1.2) without partitioning $A$ into sparse and dense parts. If the Cholesky factorization of $C$ breaks down because of a non-positive pivot, we factorize the shifted normal

Table 4 Statistics for test set 2

\begin{tabular}{llllll}
\hline Problem & $m_{s}$ & $n$ & $n n z\left(A_{s}\right)$ & $\operatorname{rdensity}\left(A_{s}\right)$ & $\operatorname{density}\left(C_{s}\right)$ \\
\hline IG5-15 & 11,369 & 6146 & 323,509 & $1.95 \times 10^{-2}$ & $1.52 \times 10^{-1}$ \\
psse0 & 26,722 & 11,028 & 102,432 & $3.63 \times 10^{-4}$ & $5.88 \times 10^{-4}$ \\
graphics & 29,493 & 11,822 & 117,954 & $3.38 \times 10^{-4}$ & $5.91 \times 10^{-4}$ \\
WORLD & 67,147 & 34,506 & 198,883 & $4.64 \times 10^{-4}$ & $4.89 \times 10^{-4}$ \\
STAT96V3 & $1,113,780$ & 33,841 & $3,317,736$ & $3.55 \times 10^{-4}$ & $3.58 \times 10^{-4}$ \\
STORMG21K & $1,377,306$ & 526,185 & $3,459,881$ & $1.93 \times 10^{-3}$ & $3.00 \times 10^{-4}$ \\
GL7d20 & $1,911,124$ & $1,437,546$ & $29,893,084$ & $2.99 \times 10^{-5}$ & $2.23 \times 10^{-4}$ \\
CONT11_L & $1,961,394$ & $1,468,599$ & $5,382,999$ & $4.77 \times 10^{-6}$ & $8.38 \times 10^{-6}$ \\
LargeRegFile & $2,111,154$ & 801,374 & $4,944,201$ & $4.99 \times 10^{-6}$ & $9.93 \times 10^{-6}$ \\
relat9 & $9,746,232$ & 274,667 & $38,955,420$ & $1.46 \times 10^{-5}$ & $5.09 \times 10^{-4}$ \\
\hline
\end{tabular}

$m_{s}, n$ and $n n z\left(A_{s}\right)$ are the number of row and column and nonzeros in $A_{s}$. $\operatorname{rdensity}\left(A_{s}\right)$ is the largest ratio of number of nonzeros in a row of $A_{s}$ to $n$ over all rows, and density $\left(C_{s}\right)$ is the ratio of the number of entries in $C_{s}$ to $n^{2}$ 
Table 5 Results for test set 1 of running the Cholesky direct solver HSL_MA87 on the normal equations (without exploiting dense rows), using LSMR for refinement

\begin{tabular}{llllllll}
\hline Identifier & $m$ & $n$ & $n n z(L)$ & Its & $T_{f}$ & $T_{s}$ & $T_{\text {total }}$ \\
\hline Trec14 & 15,904 & 3159 & $4.85 \times 10^{6}$ & 1 & 4.79 & 0.03 & 4.82 \\
Maragal_6 & 21,251 & 10,144 & $4.96 \times 10^{7}$ & 3 & 13.1 & 0.12 & 13.2 \\
Maragal_7 & 46,845 & 26,525 & $1.43 \times 10^{8}$ & 4 & 37.8 & 0.40 & 38.2 \\
SCsd8-2r & 60,550 & 8650 & $1.20 \times 10^{7}$ & 0 & 0.88 & 0.00 & 0.88 \\
PDE1 & 271,792 & 270,595 & - & - & - & - & - \\
12month1 & 872,622 & 12,471 & $7.27 \times 10^{7}$ & 1 & 42.5 & 0.35 & 42.9 \\
\hline
\end{tabular}

$n n z(L)$ denotes the number of entries in the Cholesky factor $L$ of $C$ and Its Its is the number of LSMR iterations. $T_{f}, T_{s}$ and $T_{\text {total }}$ denote the times (in seconds) to compute the normal matrix and factorize it, to run LSMR and the total time. - denotes unable to form normal matrix $C$

matrix $C+\alpha I_{n}=L(\alpha) L(\alpha)^{T}$ and use the factors as a preconditioner for the iterative method LSMR [13] (see [44]). In our tests, we set $\alpha=10^{-12}$.

Results are given in Tables 5 and 6 for the normal equations and Schur complement approaches, respectively. For problem $\mathrm{PDE} 1$, the number of entries in the normal matrix $C$ exceeds huge (1) so we cannot form $C$ and use the direct solver HSL_MA87. The reported times $T_{f}$ and $T_{p}$ for computing the Cholesky factorization of $C$ (Table 5) and the block factorization preconditioner (Table 6) include the time to form $C$ and $C_{s}$, respectively. For problem 12month1, forming $C$ (or $C_{s}$ )

Table 6 Results for test set 1 of solving the reduced augmented system (1.6) using the Schur complement approach and the Cholesky direct solver HSL_MA87. Results are also given for the indefinite solver HSL_MA9 7

\begin{tabular}{lllllllllll}
\hline Identifier & $\rho$ & $m_{d}$ & density $\left(C_{s}\right)$ & $n n z(L)$ & Its & $T_{p}$ & $T_{s}$ & $T_{\text {total }}$ & $n n z\left(L_{K}\right)$ & $T_{K}$ \\
\hline Trec14 & 0.050 & 4467 & $6.17 \times 10^{-1}$ & $1.48 \times 10^{7}$ & 1 & 4.09 & 0.06 & 4.14 & $7.97 \times 10^{6}$ & 7.18 \\
& 0.100 & 2664 & $8.31 \times 10^{-1}$ & $8.50 \times 10^{6}$ & 1 & 2.48 & 0.04 & 2.52 & $7.13 \times 10^{6}$ & 6.59 \\
& 0.200 & 1234 & $9.09 \times 10^{-1}$ & $5.73 \times 10^{6}$ & 1 & 2.41 & 0.02 & 2.43 & $6.76 \times 10^{6}$ & 5.63 \\
Maragal_6 & 0.001 & 2923 & $6.22 \times 10^{-4}$ & $4.39 \times 10^{6}$ & 3 & 1.78 & 0.16 & 1.94 & $1.78 \times 10^{7}$ & 2.54 \\
& 0.010 & 823 & $1.93 \times 10^{-2}$ & $2.46 \times 10^{7}$ & 3 & 2.77 & 0.12 & 2.88 & $2.95 \times 10^{7}$ & 5.58 \\
& 0.100 & 68 & $5.49 \times 10^{-2}$ & $4.30 \times 10^{7}$ & 3 & 4.61 & 0.15 & 4.76 & $4.15 \times 10^{7}$ & 18.3 \\
Maragal_7 & 0.001 & 4668 & $2.15 \times 10^{-4}$ & $1.12 \times 10^{7}$ & 4 & 9.51 & 0.69 & 10.2 & $6.15 \times 10^{7}$ & 12.9 \\
& 0.005 & 687 & $9.02 \times 10^{-3}$ & $9.04 \times 10^{7}$ & 3 & 11.7 & 0.36 & 12.1 & $1.10 \times 10^{8}$ & 26.5 \\
sCsd8-2r & 0.050 & 50 & $1.44 \times 10^{-3}$ & $9.20 \times 10^{4}$ & 3 & 0.03 & 0.00 & 0.03 & $5.77 \times 10^{5}$ & 0.03 \\
& 0.100 & 40 & $1.39 \times 10^{-2}$ & $5.40 \times 10^{6}$ & 3 & 0.30 & 0.04 & 0.34 & $5.25 \times 10^{6}$ & 0.29 \\
PDE1 & 0.100 & 1 & $4.52 \times 10^{-5}$ & $2.04 \times 10^{7}$ & 0 & 1.24 & 0.03 & 1.27 & $2.07 \times 10^{7}$ & 5.02 \\
12month1 & 0.050 & 3641 & $5.66 \times 10^{-1}$ & $7.74 \times 10^{7}$ & 3 & 49.3 & 0.70 & 50.0 & $8.91 \times 10^{7}$ & 61.4 \\
& 0.100 & 284 & $6.56 \times 10^{-1}$ & $7.23 \times 10^{7}$ & 3 & 42.1 & 0.54 & 42.7 & $7.53 \times 10^{7}$ & 70.0 \\
\hline
\end{tabular}

$\rho$ is the row density parameter. density $\left(C_{s}\right)$ is the ratio of the number of entries in the reduced normal matrix $C_{s}$ to $n^{2}, n n z(L)$ is total number of entries in the factors (that is, $n n z\left(L_{s}\right)+m_{d}\left(m_{d}+1\right) / 2$ ), Its is the number of GMRES iterations. $T_{p}, T_{s}$ and $T_{\text {total }}$ denote the times (in seconds) to compute the preconditioner, to run GMRES and the total time. $T_{K}$ is the time to form the reduced augmented matrix and solve using the sparse symmetric indefinite direct solver HSL_MA97 and $n n z\left(L_{K}\right)$ is the number of entries in HSL_MA97 factors 
accounts for approximately half the total time. Note we could try to employ a solver that avoids storing $C$ in main memory. The out-of-core solver HSL_MA77 [39] only requires one column of $C$ at a time and both matrix and factor data are written to files on disk, thus minimizing memory requirements. However, HSL_MA77 is for sparse matrices and when $n$ is large and $C$ is dense the amount of in-core memory available is still exceeded.

The results reported in Table 6 illustrate that the Schur complement approach is successful but to achieve savings compared to using the normal equations in terms of the size of the factors and/or the computation time, $m_{d}$ must be small compared to $n$ and the reduced normal matrix $C_{s}$ must be sparse. For the Maragal problems, we are able to choose the density $\rho$ to achieve this. In Table 6 , we include the time $T_{K}$ to form the reduced normal matrix $K$ and then factorize and solve (1.6) using the symmetric indefinite solver HSL_MA97; we also report the number $n n z\left(L_{K}\right)$ of entries in the HSL_MA97 factors of $\bar{K}$. A comparison of the times in the $T_{\text {total }}$ and $T_{K}$ columns illustrates the savings offered by the Schur complement approach that result from being able to exploit a Cholesky solver. Observe that although the symmetric indefinite solver HSL_MA97 ignores the block structure of $K$, as already noted, the sparsity-preserving nested dissection ordering it computes prior to the numerical factorization orders the dense rows last and thus the difference between $n n z(L)$ and $n n z\left(L_{K}\right)$ is generally relatively small. Furthermore, HSL_MA97 is able to take advantage of any zeros in the "dense" rows. If the number $m_{d}$ of dense rows is not small, $n n z(L)$ is dominated by the storage needed for the dense factors of $S_{d}(2.3)$ and $n n z(L)$ can then exceed $n n z\left(L_{K}\right)$; this is illustrated by problem Trec14.

\section{Iterative method results}

A software package HSL_MI35 that implements the limited memory IC algorithm outlined in Section 2.4 for computing a preconditioner for the normal equations has been developed for the HSL library. We employ this package in our experiments. Note that it handles ordering for sparsity and scaling and also automatically selects the shift $\alpha$. We use the default settings and set the parameters lsize and rsize that control the maximum number of entries in each column of the factors to 20 (see [45] for more details of the parameters).

Before we present results for our two test sets, we illustrate that, for an iterative solver, handling null columns using the approach from Lemma 2.1 can be less efficient than using a global shift when constructing the preconditioner. Results are given in Table 7 for problem scagr 7-2b from the University of Florida Collection ( $m=13,247, n=9,743$ ). This example has a single row with a density greater than $10 \%$ (this row has a density of $18.4 \%$ ). It was chosen because it illustrates how the number $n_{2}$ of null columns can increase as the number $m_{d}$ of rows that are classified as dense increases. With $\rho>0$, no shift is needed when computing the IC factorization but for $\rho=0$, the shift that is automatically selected by HSL_MI35 is $\alpha=4.1$ and preconditioned GMRES requires 370 iterations. With $\rho=0.001$, $m_{d}=263$ rows are classified as dense and there are $n_{2}=257$ null columns in $A_{s}$. For each solve, only 2 GMRES iterations are required but as there are 257 solves to 
Table 7 Results for problem scagr 7-2b of solving the reduced augmented system (1.6) using the Schur complement approach and restarted GMRES with the block IC factorization preconditioner

\begin{tabular}{lllll}
\hline$\rho$ & 0 & 0.1 & 0.01 & 0.001 \\
\hline$m_{d}$ & 0 & 1 & 7 & 263 \\
$n_{2}$ & 0 & 1 & 1 & 257 \\
density $\left(C_{s}\right)$ & $4.15 \times 10^{-2}$ & $1.45 \times 10^{-2}$ & $7.13 \times 10^{-4}$ & $5.74 \times 10^{-4}$ \\
$T_{p}$ & 0.48 & 0.50 & 0.03 & 0.10 \\
$T_{S}($ Its $)$ for $z$ & $0.33(370)$ & $0.09(99)$ & $0.004(3)$ & $0.007(2)$ \\
$T_{S}($ Its $)$ for $W$ & & $0.06(73)$ & $0.002(2)$ & $1.65(514)$ \\
$T_{\text {total }}$ & 0.81 & 0.65 & 0.04 & 1.75 \\
\hline
\end{tabular}

$\rho$ is the row density parameter. For $\rho>0$, null columns are handled using (2.10) and (2.13); $z$ and $W$ refer to (2.10). $m_{d}$ and $n_{2}$ are, respectively, the number of dense rows and the number of null columns in $A_{s} . T_{p}, T_{s}$ and $T_{\text {total }}$ denote the times (in seconds) to compute the preconditioner, to run GMRES and the total time. Its denotes the number of iterations

compute the solution $W$ of (2.10), there is a total of 514 iterations, which dominate the total cost. If we ignore the null columns, then for $\rho=0.001$, HSL_MI35 selects a global shift of $9.67 \times 10^{-7}$, GMRES then requires two iterations and the total time reduces from 1.75 to $0.12 \mathrm{~s}$. Based on this and experiments with other matrices, in the remainder of this section, we handle null columns in $A_{s}$ using a global shift.

\subsection{Results for test set 1}

Table 8 presents results for running LSMR on (1.1) using the IC preconditioner. Here, the density of the rows is ignored. In Table 9, results are given for running GMRES on the reduced augmented system using the block IC factorization preconditioner. The time $T_{p}$ includes the time for forming the reduced normal matrix $C_{s}$ and computing its IC factorization, for solving (2.18), and for forming and factorizing the Schur complement matrix (2.19). For problems Trec14, scsd8-2r and 12month1, results are given for more than one value of the parameter $\rho$ that controls which rows

Table 8 Results for test set 1 of running LMSR on (1.1) with the HSL_MI35 IC preconditioner

\begin{tabular}{llllllll}
\hline Identifier & $m$ & $n$ & $\alpha$ & Its & $T_{p}$ & $T_{s}$ & $T_{\text {total }}$ \\
\hline Trec14 & 15,904 & 3159 & $1.638 \times 10^{1}$ & 1050 & 4.52 & 3.19 & 7.71 \\
Maragal_6 & 21,251 & 10,144 & $5.120 \times 10^{-1}$ & 1130 & 6.41 & 1.59 & 8.00 \\
Maragal_7 & 46,845 & 26,525 & 2.048 & 410 & 19.9 & 1.47 & 21.4 \\
scsd8-2r & 60,550 & 8650 & $3.277 \times 10^{1}$ & 140 & 0.46 & 0.19 & 0.64 \\
PDE1 & 271,792 & 270,595 & - & - & - & - & - \\
12month1 & 872,622 & 12,471 & 1.024 & 200 & 32.6 & 10.0 & 42.7 \\
\hline
\end{tabular}

$\alpha$ denotes the global shift, Its is the number of LSMR iterations. $T_{p}, T_{s}$ and $T_{\text {total }}$ denote the times (in seconds) to compute the IC preconditioner, to run LSMR and the total time 
Table 9 Results for test set 1 of solving the reduced augmented system (1.6) using the Schur complement approach and restarted GMRES with the block IC factorization preconditioner

\begin{tabular}{lllllllllll}
\hline Identifier & $m$ & $n$ & $\rho$ & $m_{d}$ & density $\left(C_{s}\right)$ & $\alpha$ & Its & $T_{p}$ & $T_{s}$ & $T_{\text {total }}$ \\
\hline Trec14 & \multirow{2}{*}{15,904} & \multirow{2}{*}{3159} & 0.050 & 4467 & $6.17 \times 10^{-1}$ & $6.400 \times 10^{-2}$ & 163 & 2.72 & 4.17 & 6.89 \\
& & & 0.100 & 2664 & $8.31 \times 10^{-1}$ & $2.560 \times 10^{-1}$ & 245 & 1.41 & 3.49 & 4.90 \\
& & & 0.200 & 1234 & $9.09 \times 10^{-1}$ & 1.024 & 352 & 1.71 & 2.74 & 4.45 \\
Maragal_6 & 21,251 & 10,144 & 0.001 & 2923 & $6.22 \times 10^{-4}$ & $1.562 \times 10^{-5}$ & 62 & 1.46 & 1.86 & 3.33 \\
Maragal_7 & 46,845 & 26,525 & 0.001 & 4668 & $2.15 \times 10^{-4}$ & $2.500 \times 10^{-4}$ & 15 & 8.95 & 1.74 & 10.7 \\
scsd8-2r & 60,550 & 8650 & 0.050 & 50 & $1.44 \times 10^{-3}$ & $9.766 \times 10^{-7}$ & 2 & 0.03 & 0.00 & 0.03 \\
& & & 0.100 & 40 & $1.39 \times 10^{-2}$ & $3.227 \times 10^{1}$ & 68 & 0.32 & 0.13 & 0.44 \\
PDE1 & 27,1792 & 270,595 & 0.100 & 1 & $4.52 \times 10^{-5}$ & $8.000 \times 10^{-3}$ & 174 & 1.25 & 4.19 & 5.44 \\
12month1 & 872,622 & 12,471 & 0.050 & 3641 & $5.66 \times 10^{-1}$ & 1.024 & 127 & 32.8 & 13.0 & 45.8 \\
& & & 0.100 & 284 & $6.56 \times 10^{-1}$ & 1.024 & 151 & 36.1 & 10.3 & 46.4 \\
\hline
\end{tabular}

$\operatorname{density}\left(C_{s}\right)$ is the ratio of the number of entries in the reduced normal matrix $C_{s}$ to $n^{2}, \alpha$ denotes the global shift, Its is the number of GMRES iterations. $T_{p}, T_{s}$ and $T_{\text {total }}$ denote the times (in seconds) to compute the preconditioner, to run GMRES and the total time

are classified as dense. As the density of $C_{s}$ increases, a larger shift $\alpha$ is needed to prevent breakdown of the IC factorization and this has the effect of decreasing the quality of the preconditioner. However, for small $\rho$, for examples 12 month 1 and Trec14, $m_{d}$ is large and each application of the preconditioner is relatively expensive. Consequently, although the GMRES iteration count is much less than the LSMR count, for these two problems, the Schur complement approach offers no significant benefit in terms of total time. For the other problems, exploiting the dense rows is advantageous. In particular, PDE1 could not be solved using preconditioned LSMR on (1.1) but the reduced augmented system approach performs well. We observe that for the rank-deficient Maragal problems, we found it was necessary to use a very small $\rho$ to obtain a preconditioner that gave rapid convergence of GMRES (larger values of $\rho$ led to unacceptably slow convergence). Finally, we remark that the size of the incomplete factors for the normal equations approach is approximately $l$ size $* n$ while for the Schur complement approach, it is lsize $* n+m_{d}\left(m_{d}+1\right) / 2$ (recall in our experiments the HSL_MI35 memory parameter lsize is set to 20).

\subsection{Results for test set 2}

We now look at adding rows to the examples in test set 2 . We first append a single row $\left(m_{d}=1\right)$ of increasing density and solve using LMSR on (1.1) with the HSL_MI35 IC preconditioner. In Table 10, we report results for $\rho=0.01,0.1,0.5$. Problem CONT11_L is omitted because the time to compute the IC factorization exceeds $600 \mathrm{~s}$. In Table 11, results are given for $\rho=1$; results are also given for running GMRES on the reduced augmented system using the block IC factorization preconditioner. We see that, if the normal equations are used, as $\rho$ and hence the density of $C$ increases, so too do the shift $\alpha$ needed to prevent breakdown, the 
Table 10 Results for test set 2 with a single dense row of density $\rho$ appended

\begin{tabular}{|c|c|c|c|c|c|c|}
\hline Problem & $\operatorname{density}(C)$ & $\alpha$ & Its & $T_{p}$ & $T_{s}$ & $T_{\text {total }}$ \\
\hline \multicolumn{7}{|l|}{$\rho=0.01$} \\
\hline IG5-15 & $1.52 \times 10^{-1}$ & $5.120 \times 10^{-1}$ & 280 & 0.18 & 0.29 & 0.47 \\
\hline psseo & $5.33 \times 10^{-4}$ & $2.500 \times 10^{-4}$ & 1030 & 0.09 & 0.92 & 1.00 \\
\hline graphics & $6.56 \times 10^{-4}$ & $2.500 \times 10^{-4}$ & 6350 & 0.03 & 6.30 & 6.33 \\
\hline WORLD & $5.88 \times 10^{-4}$ & $1.638 \times 10^{1}$ & 1340 & 0.28 & 3.57 & 3.85 \\
\hline STAT96V3 & $4.57 \times 10^{-4}$ & 1.024 & 560 & 0.27 & 12.1 & 12.4 \\
\hline STORMG21K & $4.00 \times 10^{-4}$ & $1.311 \times 10^{2}$ & 1620 & 51.9 & 101 & 153 \\
\hline GL7d20 & $3.23 \times 10^{-4}$ & $5.243 \times 10^{2}$ & 40 & 166 & 19.1 & 185 \\
\hline LargeRegFile & $1.10 \times 10^{-4}$ & $1.311 \times 10^{2}$ & 70 & 127 & 7.68 & 134 \\
\hline relat 9 & $6.09 \times 10^{-4}$ & $1.311 \times 10^{2}$ & 90 & 19.8 & 29.1 & 48.9 \\
\hline \multicolumn{7}{|l|}{$\rho=0.1$} \\
\hline IG5-15 & $1.61 \times 10^{-1}$ & $3.277 \times 10^{1}$ & 810 & 0.23 & 0.83 & 1.07 \\
\hline psseo & $1.06 \times 10^{-2}$ & $3.277 \times 10^{1}$ & 38,200 & 0.22 & 40.2 & 40.4 \\
\hline graphics & $1.06 \times 10^{-2}$ & $3.277 \times 10^{1}$ & $>100,000$ & 0.23 & - & - \\
\hline WORLD & $1.05 \times 10^{-2}$ & $1.311 \times 10^{2}$ & 1840 & 0.88 & 4.91 & 5.79 \\
\hline STAT96V3 & $1.04 \times 10^{-2}$ & $1.311 \times 10^{2}$ & 880 & 1.19 & 19.86 & 21.0 \\
\hline STORMG $21 \mathrm{~K}$ & $1.03 \times 10^{-2}$ & $1.049 \times 10^{3}$ & 1470 & 192 & 97.8 & 290 \\
\hline GL $7 d 20$ & - & - & - & $>600$ & - & $>600$ \\
\hline LargeRegFile & - & - & - & $>600$ & - & $>600$ \\
\hline relat 9 & $1.05 \times 10^{-2}$ & $5.243 \times 10^{2}$ & 90 & 63.7 & 28.6 & 92.3 \\
\hline \multicolumn{7}{|l|}{$\rho=0.5$} \\
\hline IG5-15 & $3.64 \times 10^{-1}$ & $6.554 \times 10^{1}$ & 880 & 0.50 & 0.91 & 1.41 \\
\hline psseo & $2.50 \times 10^{-1}$ & $2.621 \times 10^{2}$ & 34,570 & 1.31 & 40.64 & 41.96 \\
\hline graphics & $2.50 \times 10^{-1}$ & $2.621 \times 10^{2}$ & $>100,000$ & 1.38 & - & - \\
\hline WORLD & $2.50 \times 10^{-1}$ & $5.243 \times 10^{2}$ & 2020 & 13.10 & 6.42 & 19.52 \\
\hline STAT96V3 & $2.50 \times 10^{-1}$ & $5.243 \times 10^{2}$ & 760 & 10.91 & 17.03 & 27.94 \\
\hline STORMG21K & - & - & - & $>600$ & - & $>600$ \\
\hline GL7d20 & - & - & - & $>600$ & - & $>600$ \\
\hline LargeRegFile & - & - & - & $>600$ & - & $>600$ \\
\hline relat 9 & - & - & - & $>600$ & - & $>600$ \\
\hline
\end{tabular}

Results are for LMSR on (1.1) using the IC factorization preconditioner. $\alpha$ denotes the global shift, Its is the number of iterations. $T_{p}, T_{s}$ and $T_{\text {total }}$ denote the times (in seconds) to compute the IC preconditioner, to run the iterative solver and the total time. - indicates statistic unavailable

time to compute the IC factorization, and the iterations for convergence. Indeed, for the large examples, the time exceeds our limit of $600 \mathrm{~s}$. By contrast, for preconditioned GMRES on the reduced augmented system, the shift and the times to compute the incomplete factorization and achieve convergence are essentially independent of $\rho$ (and for this reason only results for $\rho=1.0$ are included in Table 11). Furthermore, this approach uses a smaller shift than for the normal equations and 
Table 11 Results for test set 2 with a single dense row $(\rho=1.0)$ appended

\begin{tabular}{|c|c|c|c|c|c|c|c|c|c|c|}
\hline \multirow[t]{2}{*}{ Problem } & \multicolumn{5}{|c|}{ Preconditioned LSMR } & \multicolumn{5}{|c|}{$\begin{array}{l}\text { Reduced augmented system with } \\
\text { GMRES }\end{array}$} \\
\hline & $\alpha$ & Its & $T_{p}$ & $T_{s}$ & $T_{\text {total }}$ & $\alpha$ & Its & $T_{p}$ & $T_{s}$ & $T_{\text {total }}$ \\
\hline IG5-15 & $6.5536 \times 10^{1}$ & 810 & 0.92 & 0.82 & 1.73 & 1.024 & 337 & 0.47 & 1.08 & 1.55 \\
\hline psseo & $2.6214 \times 10^{2}$ & 33,690 & 2.06 & 39.8 & 41.9 & 0.0 & 81 & 0.01 & 0.05 & 0.06 \\
\hline graphics & $2.6214 \times 10^{2}$ & $>100,000$ & 3.56 & $>134$ & $>138$ & $9.766 \times 10^{-7}$ & 900 & 0.02 & 1.49 & 1.51 \\
\hline WORLD & $5.2429 \times 10^{2}$ & 2040 & 39.9 & 7.16 & 47.1 & $1.280 \times 10^{-1}$ & 294 & 1.32 & 1.31 & 2.63 \\
\hline STAT96V3 & $5.2429 \times 10^{2}$ & 750 & 23.9 & 16.8 & 40.7 & 0.0 & 20 & 0.20 & 0.04 & 0.23 \\
\hline STORMG21K & - & - & $>600$ & - & $>600$ & $2.621 \times 10^{2}$ & 1320 & 19.5 & 211 & 230 \\
\hline GL7d20 & - & - & $>600$ & - & $>600$ & $5.120 \times 10^{-1}$ & 32 & 187 & 39.0 & 226 \\
\hline CONT11_L & - & - & $>600$ & - & $>600$ & $8.000 \times 10^{-3}$ & 142 & 9.23 & 22.8 & 32.0 \\
\hline LargeRegFile & - & - & $>600$ & - & $>600$ & 0.0 & 11 & 1.61 & 0.53 & 2.14 \\
\hline relat 9 & - & - & $>600$ & - & $>600$ & $9.766 \times 10^{-7}$ & 41 & 32.3 & 3.18 & 35.5 \\
\hline
\end{tabular}

Results are for LMSR on (1.1) using the IC factorization preconditioner and for solving the reduced augmented system (1.6) using the Schur complement approach and restarted GMRES with the block IC factorization preconditioner. $\alpha$ denotes the global shift, Its is the number of iterations. $T_{p}, T_{s}$ and $T_{\text {total }}$ denote the times (in seconds) to compute the IC preconditioner, to run the iterative solver and the total time. - indicates statistic unavailable

produces a much higher quality preconditioner, leading to significantly faster times. With more than one added row, the density of $C$ often increases further, making the normal equation approach even less feasible. For the augmented approach, adding

Table 12 Results for test set 2 when $m_{d}=1,50$ and 100 rows are appended

\begin{tabular}{|c|c|c|c|c|c|c|c|c|c|c|c|c|}
\hline \multirow[t]{3}{*}{ Problem } & \multicolumn{6}{|c|}{ GMRES } & \multicolumn{6}{|c|}{ MINRES } \\
\hline & \multicolumn{2}{|c|}{$m_{d}=1$} & \multicolumn{2}{|c|}{$m_{d}=50$} & \multicolumn{2}{|c|}{$m_{d}=100$} & \multicolumn{2}{|c|}{$m_{d}=1$} & \multicolumn{2}{|c|}{$m_{d}=50$} & \multicolumn{2}{|c|}{$m_{d}=100$} \\
\hline & Its & $T_{\text {total }}$ & Its & $T_{\text {total }}$ & Its & $T_{\text {total }}$ & Its & $T_{\text {total }}$ & Its & $T_{\text {total }}$ & Its & $T_{\text {total }}$ \\
\hline IG5-15 & 337 & 1.55 & 151 & 0.95 & 129 & 0.91 & 427 & 1.06 & 554 & 1.48 & 480 & 1.21 \\
\hline psise0 & 81 & 0.06 & 40 & 0.05 & 30 & 0.07 & 117 & 0.16 & 202 & 0.25 & 210 & 0.31 \\
\hline graphics & 900 & 1.51 & 107 & 0.12 & 68 & 0.11 & 841 & 0.66 & 517 & 0.45 & 389 & 0.46 \\
\hline WORLD & 294 & 2.63 & 158 & 2.13 & 116 & 2.21 & 348 & 2.35 & 563 & 3.77 & 463 & 4.48 \\
\hline STAT96V3 & 20 & 0.23 & 7 & 0.25 & 7 & 0.32 & 31 & 0.52 & 50 & 0.81 & 55 & 1.04 \\
\hline STORMG21K & 1320 & 230 & 906 & 194 & 959 & 228 & 6197 & 335 & - & $>600$ & - & $>600$ \\
\hline GL7d20 & 32 & 226 & 22 & 222 & 22 & 231 & 36 & 207 & 60 & 232 & 60 & 241 \\
\hline CONT11_L & 142 & 32.0 & 12 & 13.0 & 13 & 15.9 & 173 & 26.6 & 1556 & 290 & 1455 & 395 \\
\hline LargeRegFile & 11 & 2.14 & 9 & 3.28 & 9 & 4.83 & 16 & 2.71 & 23 & 4.86 & 22 & 6.58 \\
\hline relat9 & 41 & 35.5 & 160 & 33.3 & 279 & 65.1 & 54 & 56.7 & 726 & 379 & - & $>600$ \\
\hline
\end{tabular}

The reduced augmented system (1.6) is solved using the Schur complement approach and restarted GMRES and MINRES with the block IC factorization preconditioner. Its is the number of iterations and $T_{\text {total }}$ is the total time 
more than one row does not affect $C_{s}$ or the time to compute the incomplete factorization but does result in the dense factorization of the Schur complement matrix becoming more expensive. For most of our test problems, the number of GMRES iterations decreases as the number of added rows increases (for example, psse 0 and graphics) but for others (including relat9), the converse is true (see Table 12). In these tests, MINRES was uncompetitive compared to GMRES in terms of iteration counts and times but has the advantage of requiring less storage. We remark that for problem STORMG21K, the iteration counts are high. For this example, the shift $\alpha$ that is needed by the IC factorization is large, which negatively effects the quality of the resulting preconditioner.

\section{Concluding remarks}

In this paper, we have focused on using the Schur complement approach to solve large-scale linear least-squares problems in which the system matrix $A$ contains a number of nearly dense rows. Our proposed approach involves using a regularization parameter and then applying a Cholesky solver to the shifted reduced normal equations. A small number of steps of the iterative solver GMRES applied to the reduced augmented system are then employed to recover the solution of the original (unshifted) problem. We have considered some hard-to-solve problems (including some rank-deficient examples) from practical applications and shown that this approach offers savings (in terms of time and the size of the factors) compared to using a general sparse symmetric indefinite solver. The approach can be used with an incomplete Cholesky factorization preconditioner. In this case, a larger shift is required to prevent breakdown of the factorization, and this increases with the density of the reduced normal matrix and leads to a greater number of iterations being needed for convergence.

In addition to problems in which $A$ contains some dense rows, we have considered examples where we added a number of dense rows to $A$. We found that, if the appended dense rows were not explicitly exploited, in some cases we were unable to achieve acceptable convergence using IC preconditioned LMSR. However, the use of the reduced normal matrix reduces the size of the shift that is needed, resulting in a higher quality preconditioner that successfully solved these examples.

We note that this paper complements the recent work in [48] in which preconditioned conjugate gradients (CGLS) is the main iterative method. It is shown there that once the dense rows are clearly defined and detected, the preconditioned iterative method is able to solve the problem extremely efficiently. In this study, we have gone beyond preconditioned conjugate gradients and use the power of direct methods to extend the solution of mixed sparse-dense problems to tough problems such as WORLD and the Maragal matrices that have so many null columns that further research is needed to be able to treat them as in [48].

Finally, we remark that although our main motivation for partitioning $A$ is the presence of one or more dense rows, there are other possible reasons for employing a partitioning of the form (1.3). For example, a set of additional rows, that are not necessarily dense, is obtained by repeatedly adding new data into the least-squares 
estimation of parameters in a linear model (see, for example, [3, 4]). Nowadays, there exist important applications based on this motivation related to Kalman filtering or solving recursive least-squares problems, see the seminal paper [25] or for a comprehensive introduction [10, 43]. Furthermore, additional constraints for the least-squares problem represented by $A_{d}$ and $b_{d}$ naturally arise with rank-deficient least-squares problems (for instance, $[5,7,32]$ ). If extra rows are added, the sparse (incomplete) Cholesky factorization within the Schur complement approach can be reused and so the only work needed to solve the updated system (or, in the incomplete case, to apply a preconditioner for the enlarged system) is the solution of triangular systems.

Acknowledgements We are very grateful to Michael Saunders and to an anonymous reviewer for their careful reading of our manuscript and for their constructive comments and suggestions that led to improvements in the presentation of this paper.

Open Access This article is distributed under the terms of the Creative Commons Attribution 4.0 International License (http://creativecommons.org/licenses/by/4.0/), which permits unrestricted use, distribution, and reproduction in any medium, provided you give appropriate credit to the original author(s) and the source, provide a link to the Creative Commons license, and indicate if changes were made.

\section{References}

1. Andersen, E.D., Gondzio, J., Mészáros, C., Xu, X.: Implementation of Interior Point Methods for Large Scale Linear Programming. HEC/Université de Genève (1996)

2. Andersen, K.D.: A modified Schur complement method for handling dense columns in interior point methods for linear programming (1996)

3. Anderson, O.D.: An improved approach to inverting the autocovariance matrix of a general mixed autoregressive moving average time process. Australian J. Statist. 18(1-2), 73-75 (1976)

4. Anderson, O.D.: On the inverse of the autocovariance matrix for a general moving average process. Biometrika 63(2), 391-394 (1976)

5. Avron, H., Ng, E., Toledo, S.: Using perturbed $Q R$ factorizations to solve linear least-squares problems. SIAM J. Matrix Anal. Appl. 31(2), 674-693 (2009)

6. Benzi, M.: Preconditioning techniques for large linear systems: a survey. J. Comput. Phys. 182(2), 418-477 (2002)

7. Björck, Å.: A general updating algorithm for constrained linear least squares problems. SIAM J. Sci. Statist. Comput. 5(2), 394-402 (1984)

8. Björck, Å.: Numerical Methods for Least Squares Problems. SIAM, Philadelphia (1996)

9. Davis, T.A., Hu, Y.: The University of Florida sparse matrix collection. ACM Trans. Math. Softw. 38(1), 1, 1-28 (2011)

10. Diniz, P.S.R.: Adaptive Filtering: Algorithms and Practical Implementation. Springer, 4th ed 2013 edition (2012)

11. Dollar, H.S., Scott, J.A.: A note on fast approximate minimum degree orderings for matrices with some dense rows. Numer. Linear Algebra Appl. 17, 43-55 (2010)

12. Duff, I.S.: MA57-a code for the solution of sparse symmetric definite and indefinite systems. ACM Trans. Math. Softw. 30, 118-154 (2004)

13. Fong, D.C.-L., Saunders, M.A.: LSMR: an iterative algorithm for sparse least-squares problems. SIAM J. Sci. Comput. 33(5), 2950-2971 (2011)

14. George, A., Heath, M.T.: Solution of sparse linear least squares problems using Givens rotations. Linear Algebra Appl. 34, 69-83 (1980)

15. Gill, P.E., Murray, W., Ponceleon, D.B., Saunders, M.A.: Solving reduced KKT systems in barrier methods for linear and quadratic programming. Technical Report SOL 91-7, Department of Operations Research Stanford University (1991) 
16. Gill, P.E., Saunders, M.A., Shinnerl, J.R.: On the stability of Cholesky factorization for symmetric quasidefinite systems. SIAM J. Matrix Anal. Appl. 17(1), 35-46 (1996)

17. Goldfarb, D., Scheinberg, K.: A product-form Cholesky factorization method for handling dense columns in interior point methods for linear programming. Math. Program. Series A 99, 1-34 (2004)

18. Golub, G.H., Van Loan, C.F.: Unsymmetric positive definite linear systems. Linear Algebra Appl. 28, 85-97 (1979)

19. Gould, N.I.M., Orban, D., Toint, Ph.L.: CUTEst: a constrained and unconstrained testing environment with safe threads for mathematical optimization. Comput. Optim. Appl. 60, 545-557 (2015)

20. Gould, N.I.M., Scott, J.A.: The state-of-the-art of preconditioners for sparse linear least squares problems. ACM Trans. Math. Softw. 43(4), 36,1-35 (2017)

21. Greif, C., He, S., Liu, P.: SYM-ILDL C++ package for incomplete factorizations of symmetric indefinite matrices https://github.com/inutard/matrix-factor (2013)

22. Hogg, J.D., Reid, J.K., Scott, J.A.: Design of a multicore sparse Cholesky factorization using DAGs. SIAM J. Sci. Comput. 32, 3627-3649 (2010)

23. Hogg, J.D., Scott, J.A.: HSL_MA97: A bit-compatible multifrontal code for sparse symmetric systems. Technical Report RAL-TR-2011-024 Rutherford Appleton Laboratory (2011)

24. HSL: A collection of Fortran codes for large-scale scientific computation. http://www.hsl.rl.ac.uk (2017)

25. Kalman, R.E.: A new approach to linear filtering and prediction problems. Trans. ASME-J. Basic Eng. 82(Series D), 35-45 (1960)

26. Karypis, G., Kumar, V.: METIS: software package for partitioning unstructured graphs, partitioning meshes, and computing fill-reducing orderings of sparse matrices (version 3.0). Technical report, University of Minnesota Department of Computer Science and Army HPC Research Center (1997)

27. Lin, C.-J., Moré, J.J.: Incomplete Cholesky factorizations with limited memory. SIAM J. Sci. Comput. 21(1), 24-45 (1999)

28. Lustig, I.J., Marsten, R.E., Shanno, D.F.: Computational experience with a primal-dual interior point method for linear programming. Linear Algebra Appl. 152, 191-222 (1991)

29. Manteuffel, T.A.: An incomplete factorization technique for positive definite linear systems. Math. Comput. 34, 473-497 (1980)

30. Marxen, A.: Primal barrier methods for linear programming. Technical Report SOL 89-6, Department of Operations Research Stanford University (1989)

31. MUMPS: A MUltifrontal Massively Parallel sparse direct Solver. http://graal.ens-lyon.fr/MUMPS/ (2016)

32. $\mathrm{Ng}$, E.: A scheme for handling rank-deficiency in the solution of sparse linear least squares problems. SIAM J. Sci. Statist. Comput. 12(5), 1173-1183 (1991)

33. Okulicka-Dłużewska, F., Rozložník, M., Smoktunowicz, A.: Cholesky-like factorization of symmetric indefinite matrices and orthogonalization with respect to bilinear forms. SIAM J. Matrix Anal. Appl. 36(2), 727-751 (2015)

34. Orban, D.: Limited-memory $\mathrm{LDL}^{\top}$ factorization of symmetric quasi-definite matrices with application to constrained optimization. Numer. Algor. 70(1), 9-41 (2015)

35. Orban, D., Arioli, M.: Iterative Solution of Symmetric Quasi-Definite Linear Systems, volume 3 of SIAM Spotlights Society for Industrial and Applied Mathematics. SIAM, Philadelphia (2017)

36. Paige, C.C., Saunders, M.A.: Solution of sparse indefinite systems of linear equations. SIAM J. Numer. Anal. 12(4), 617-629 (1975)

37. Paige, C.C., Saunders, M.A.: Algorithm 583; LSQR: Sparse linear equations and least-squares problems. ACM Trans. Math. Softw. 8(2), 195-209 (1982)

38. Paige, C.C., Saunders, M.A.: LSQR: an algorithm for sparse linear equations and sparse least squares. ACM Trans. Math. Softw. 8(1), 43-71 (1982)

39. Reid, J.K., Scott, J.A.: An out-of-core sparse Cholesky solver. ACM Trans. Math. Softw. 36(2), 9,1-36 (2009)

40. Saad, Y. Iterative Methods for Sparse Linear Systems, 2nd edn. SIAM, Philadelphia (2003)

41. Saad, Y., Schultz, M.H.: GMRES: a generalized minimal residual algorithm for solving nonsymmetric linear systems. SIAM J. Sci. Stat. Comput. 7, 856-869 (1986)

42. Saunders, M.A.: Cholesky-based methods for sparse least squares: The benefits of regularization. Technical Report SOL 95-1, Department of Operations Research, Stanford University, 1995. In: Adams, L., Nazareth, J.L. (eds.) Linear and Nonlinear Conjugate Gradient-Related Methods, pp. 92-100. SIAM, Philadelphia (1996) 
43. Sayed, A.H.: Fundamentals of Adaptive Filtering. Wiley (2003)

44. Scott, J.A.: On using Cholesky-based factorizations for solving rank-deficient sparse linear leastsquares problems. SIAM J. Sci. Comput. 39(4), C319-C339 (2017)

45. Scott, J.A., Tůma, M.: HSL_MI28: an efficient and robust limited-memory incomplete Cholesky factorization code. ACM Trans. Math. Softw 40(4), 24,1-19 (2014)

46. Scott, J.A., Tůma, M.: On positive semidefinite modification schemes for incomplete Cholesky factorization. SIAM J. Sci. Comput. 36(2), A609-A633 (2014)

47. Scott, J.A., Tůma, M.: On signed incomplete Cholesky factorization preconditioners for saddle-point systems. SIAM J. Sci. Comput. 36(6), A2984-A3010 (2014)

48. Scott, J.A., Tůma, M.: Solving mixed sparse-dense linear least-squares problems by preconditioned iterative methods. SIAM J. Sci. Comput. 39(6), A2422-A2437 (2017)

49. Sun, C.: Dealing with dense rows in the solution of sparse linear least squares problems. Research Report CTC95TR227, Advanced Computing Research Institute Cornell Theory Center; Cornell University (1995)

50. Sun, C.: Parallel solution of sparse linear least squares problems on distributed-memory multiprocessors. Parallel Comput. 23(13), 2075-2093 (1997)

51. Vanderbei, R.J.: Splitting dense columns in sparse linear systems. Linear Algebra Appl. 152, 107-117 (1991)

52. Vanderbei, R.J.: Symmetric quasidefinite matrices. SIAM J. Optim. 5(1), 100-113 (1995)

53. WSMP: Watson sparse matrix package (WSMP). http://researcher.watson.ibm.com/researcher/view_group. php?id=1426 (2016) 